ESTUDO TERMODINÂMICO DA APLICAÇÃO DE HIDROCARBONETOS EM SISTEMA DE REFRIGERAÇÃO

\author{
Jucélio Gomes da Silva \\ Aluno Bolsista do Curso Técnico Subsequente em Refrigeração e Climatização. Grupo de \\ Estudos em Refrigeração e Ar-Condicionado. IFRN - Campus Santa Cruz. E-mail: \\ juceliosilva2010@yahoo.com.br \\ Maria Luiza Ramos da Silva \\ Aluna Bolsista do Curso Técnico Subsequente em Refrigeração e Climatização. Grupo de \\ Estudos em Refrigeração e Ar-Condicionado. IFRN - Campus Santa Cruz. E-mail: \\ marialuiza_ramosdasilva@yahoo.com \\ Igor Marcel Gomes Almeida \\ Professor do Instituto Federal de Educação, Ciência e Tecnologia do Rio Grande do Norte. \\ Grupo de Estudos em Refrigeração e Ar-Condicionado. Campus Santa Cruz. E-mail: \\ igor.almeida@ifrn.edu.br \\ Cleiton Rubens Formiga Barbosa \\ Professor do Departamento de Engenharia Mecânica. Universidade Federal do Rio Grande \\ do Norte. E-mail: cleiton@ufrnet.br
}

\title{
RESUMO
}

Os esforços de pesquisa e desenvolvimento na área de Refrigeração e Ar-Condicionado aplicados ao uso de fluidos refrigerantes oriundos de produtos naturais não está associada somente à necessidade de preservação do meio ambiente em si, mas também apresenta grande importância devido a necessidade latente do aumento da eficiência energética dos equipamentos. Neste sentido, o presente trabalho trata da avaliação termodinâmica da aplicação de hidrocarbonetos como fluidos refrigerantes em um sistema de refrigeração residencial que utiliza o HFC134a como fluido de trabalho. Uma análise teóricocomputacional foi desenvolvida para o R134a, propano (R290) e as misturas selecionadas (R290/R600a 60\%/40\%， R290/R600a/R134a 40\%/30\%/30\% e R600a/R290-GLP $70 \% / 30 \%$ ) no ciclo de refrigeração padrão ASHRAE, utilizando as propriedades termodinâmicas e termofísicas fornecidas pelo software REFPROP 6.0. Os resultados das simulações computacionais foram comparados entre os fluidos para indicação da melhor alternativa ao HFC134a. Neste sentido, pôde-se observar que os hidrocarbonetos reduzem os níveis de pressão no condensador e evaporador, além de menores trabalhos de compressão serem necessários no sistema devido às propriedades termofísicas privilegiadas destes fluidos. A utilização destes fluidos também proporciona uma menor temperatura de descarga do compressor, incrementando a vida útil deste componente de alto valor do sistema.

PALAVRAS-CHAVE: refrigerantes naturais, análise termodinâmica, hidrocarbonetos, coeficiente de performance. 


\title{
THERMODYNAMIC STUDY OF HYDROCARBONS APPLICATION IN REFRIGERATION SYSTEM
}

\begin{abstract}
The research efforts and development in the Refrigeration and Air Conditioning sector applied to the use of natural refrigeration fluids is not associated only with the need to preserve the environment alone, but has great importance with regard to the latent need for energy efficient equipment. With this perspective, the present study deals with the thermodynamic evaluation of the use of hydrocarbons refrigerants in household refrigeration systems that utilize HFC134a as a working fluid. A theoretical-computational analysis was developed for R134a, propane (R290) and the selected mixtures (R290/R600a $60 \% / 40 \%$, R290/R600a/R134a 40\%/30\%/30\% and R600a/R290-GLP 70\%/30\%) in the standard refrigeration cycle ASHRAE, using the thermodynamic and thermophysical properties provided by the REFPROP 6.0 software. The results of computational simulations between the fluids were compared to find the evidence of the best alternative to HFC134a. In this sense, it was observed that the hydrocarbons reduced the levels of pressure on the condenser and evaporator, along with smaller compression tasks necessary in the system, owing to the thermo-physical properties privileged in these fluids. The use of these fluids is also proportionate to lower temperatures of compressor flushing, increasing the life of this highly valuable component of the system.
\end{abstract}

KEYWORDS: natural refrigerants, thermodynamic analysis, hydrocarbons, coefficient of performance. 


\section{ESTUDO TERMODINÂMICO DA APLICAÇÃO DE HIDROCARBONETOS EM SISTEMA DE REFRIGERAÇÃO}

\section{INTRODUÇÃO}

Em setembro de 2007, quando se comemoravam os 20 anos de sucessos do Protocolo de Montreal, uma nova decisão histórica foi tomada pelo conjunto dos países signatários do referido acordo. Com o fim do uso dos CFC's, previsto para 2010, decidiu-se pelo início do processo de substituição dos HCFC's já em 2013, antecipando em dez anos o prazo previsto pelo Protocolo de Montreal para o abandono do emprego destes gases. Além dos benefícios para a recomposição da Camada de Ozônio, objeto do Protocolo, a medida traz também um enorme benefício para o regime climático, dado o acentuado Potencial de Aquecimento Global (GWP) dos HCFC's.

Ao longo de seus 20 anos, a eliminação dos CFC's contribuiu significativamente para que se evitasse a emissão de bilhões de toneladas de $\mathrm{CO}_{2}$ equivalente e pode continuar a fazêlo com uma relação custo-benefício das mais favoráveis. Somente no Brasil, a antecipação do prazo de eliminação dos CFC's determinada pela Resolução 267/2000 do CONAMA evitou o consumo de 36,5 mil toneladas PDO de CFC's, o que equivale, quanto ao seu efeito para o aquecimento global, a 360 milhões de toneladas de $\mathrm{CO}_{2}$. A título de comparação, o ProÁlcool, o mais bem sucedido programa de combustíveis renováveis do mundo, evitou de 1975 a 2005 a emissão de 650 milhões de toneladas de $\mathrm{CO}_{2}$. Antecipar os prazos de abandono de HCFC's significará novos ganhos.

No entanto, os esforços de pesquisa e desenvolvimento na área de Refrigeração e ArCondicionado aplicados ao uso de fluidos refrigerantes naturais não está associada somente à necessidade de preservação do meio ambiente em si, mas também apresenta grande importância na necessidade latente do aumento da eficiência energética dos equipamentos. Tal característica é observada na Decisão XIX/6 do Protocolo de Montreal. Neste sentido, de acordo com diversas pesquisas realizadas com tais fluidos, a utilização de hidrocarbonetos em operações de drop-in (processo de substituição de um fluido refrigerante sem alteração nos componentes estruturais do refrigerador) em equipamentos de refrigeração proporciona condições favoráveis à redução do consumo energético. Hidrocarbonetos, como o gás liquefeito de petróleo (GLP), são ambientalmente corretos e seus produtos são disponíveis em abundância na natureza. Neste sentido, a utilização destas substâncias como fluidos refrigerantes em refrigeradores domésticos é muito atrativa. Pouca informação se encontra disponível na literatura científica sobre a utilização de hidrocarbonetos em refrigeradores domésticos. A maioria dos trabalhos está concentrada na substituição do CFC-12, e poucas pesquisas abordam a substituição do HFC-134a, que é o fluido atualmente utilizado nestes equipamentos.

Vale lembrar que a substituição dos HCFC's se dará com a introdução de outros gases. Caso o HCFC- 22 venha a ser substituído predominantemente pelo HFC-134a, uma das alternativas mais consolidadas no mercado, grande parte deste ganho seria anulada, já que o GWP das duas substâncias é similar.

Os refrigeradores estão dentre os equipamentos que representam as maiores participações no consumo residencial de eletricidade no Brasil. Essa participação é estimada em torno de $30 \%$ representando em 2004 um consumo de eletricidade aproximado de $23.000 \mathrm{GWh}$, cerca de $6,3 \%$ do consumo total de eletricidade no país. Apesar dos avanços no aumento da 
eficiência no consumo de energia dos refrigeradores disponibilizados no mercado, o que é indicado pelo selo PROCEL, ainda existem em operação nos domicílios brasileiros muitos equipamentos antigos com tecnologias ineficientes.

Estima-se que a participação de refrigeradores com idade superior a 15 anos no estoque em uso seja de aproximadamente $12 \%$. Isso indica prolongamento da utilização de equipamentos com utilização de tecnologias ineficientes e com eficiência degradada. $\mathrm{O}$ consumo desses equipamentos pode ser até 5 vezes superior aos equipamentos novos com classificação A do selo PROCEL (MELO e JANNUZZI, 2008).

Desta forma, observa-se a importância de buscas de alternativas para redução do consumo energético de refrigeradores domésticos no Brasil. Especificamente no caso da cidade de Santa Cruz-RN, a maior parcela de consumo energético anual se dá no setor residencial, onde os refrigeradores contribuem em grande parte com tal nível de consumo, que é de 8.695 MWh, de um total de 19.232 MWh incluindo todos os setores (industrial, comercial, rural, poderes públicos, iluminação pública e outros setores) (IBGE, 2007). A constatação desta realidade local está sendo analisada no contexto do desenvolvimento de projetos de extensão junto à comunidade rural ou urbana para levantamento do consumo energético de refrigeração e estudo da possibilidade de realização de projetos de drop-in nos equipamentos. Uma das alternativas possíveis para tal objetivo consiste na substituição de fluidos refrigerantes sintéticos por outros fluidos oriundos de produtos naturais, como os hidrocarbonetos, em operações de drop-in. Resultados experimentais demonstram reduções, no consumo de energia, de 4,3\% (MOHANRAJ et al., 2007), 5\% (WONGWISES e CHIMRES, 2005) e de até 20\% (MACLAINE-CROSS, 1997).

Um elemento chave para a transformação do mercado de refrigeradores domésticos, especificamente quanto à eficiência energética consiste no desenvolvimento de um processo de conscientização e reforço no processo educativo da população sobre o uso da energia de forma eficiente nestes equipamentos. Tal objetivo pode ser alcançado através do desenvolvimento de um currículo de ensino médio que aborde questões referentes à eficiência energética e à utilização da energia em disciplinas da área de ciências. Além do desenvolvimento deste currículo, uma outra tarefa importante consiste na divulgação de informação ao público em geral. Meios de comunicação social podem auxiliar na sensibilização de consumidores e usuários de sistemas de refrigeração sobre a utilização eficiente da energia. Documentários públicos e programas de educação são outros componentes essenciais na campanha para a transformação do mercado (VAN BUSKIRK et al., 2007).

Desta forma, tal projeto de pesquisa visa a disseminação de informações acerca do tema da eficiência energética aplicada ao setor da refrigeração residencial junto aos alunos do IFRN - Campus Santa Cruz, além do desenvolvimento de projetos de extensão junto à comunidade do Trairi como forma de conscientização sobre o uso eficiente da energia e aspectos relacionados a políticas energéticas na região. Em países e regiões em desenvolvimento, iniciativas de transformação do mercado para melhoria da eficiência energética em aplicações residenciais mostraram grandes benefícios MEYERS et al., 2003).

O presente artigo constitui parte introdutória de projeto de pesquisa relacionado ao uso de hidrocarbonetos em sistemas de refrigeração residencial. Enfoca-se a análise termodinâmica da utilização destes fluidos refrigerantes em comparação aos fluidos 
sintéticos convencionais através dos resultados de simulações computacionais realizadas mediante utilização do software REFPROP 6.0 (McLINDEN et al., 1998), de avaliação de propriedades termodinâmicas e termofísicas de fluidos refrigerantes.

Uma análise teórica foi desenvolvida para o R134a, propano (R290) e as misturas selecionadas (R290/R600a 60\%/40\%, R290/R600a/R134a 40\%/30\%/30\% e R600a/R290GLP 70\%/30\%) no ciclo de refrigeração padrão ASHRAE (temperatura de evaporação: $-23,3^{\circ} \mathrm{C}$, temperatura de condensação: $54,4^{\circ} \mathrm{C}$, temperatura de líquido e de sucção: $32,2^{\circ} \mathrm{C}$ ), utilizando as propriedades termodinâmicas do REFPROP 6.0, conforme recomendado por KIM et al. (1998).

\section{REVISÃO BIBLIOGRÁFICA}

Com a demonstração da relação existente entre as emissões de CFC's e a destruição da camada de ozônio estratosférico feita por Rowland e Molina em 1974 e a consequente assinatura em 1987, do Protocolo de Montreal em 1987, que impôs metas para a eliminação dos CFC's e HCFC's, a questão do impacto ambiental causado pela indústria de refrigeração e climatização parecia então resolvida. Entretanto, a discussão sobre o aquecimento global, que há algum tempo vinha ganhando força na comunidade científica, findou por levar, em 1997, à assinatura do Tratado de Kyoto, onde se assumiram metas para a redução da emissão de gases do efeito estufa (entre eles os HFC's) pelos países desenvolvidos. Novamente, a comunidade industrial e científica atuando no setor de refrigeração e climatização mundial se encontrava em face da necessidade de desenvolver uma nova geração de fluidos refrigerantes, agora comprometidos também com a preservação do meio-ambiente.

RICHARDSON e BUTTERWORTH (1995) conduziram experimentos para investigar a performance de refrigerantes hidrocarbonetos em um sistema de refrigeração por compressão de vapor hermético. Os testes foram feitos utilizando R12, propano e misturas de propano/isobutano variando em torno da composição de 50\%/50\%. Os autores constataram que a mistura de propano/isobutano com composição de $50 \%$ em massa de propano apresenta maior COP, menor carga no sistema e menor temperatura na parede do compressor quando comparado ao R12.

MACLAINE-CROSS (1997) demonstrou que os refrigerantes hidrocarbonetos proporcionam $20 \%$ de redução no consumo de energia em relação ao CFC12 e HFC134a.

KIM et al., (1998) avaliaram a performance do refrigerante hidrocarboneto R600a como alternativa ao R12 em um refrigerador doméstico de 215 litros de capacidade de degelo automático. Os autores desenvolveram uma análise teórica utilizando o software REFPROP, baseada no ciclo de refrigeração por compressão de vapor padrão ASHRAE, e em seguida conduziram uma série de testes experimentais com o R600a. Para a realização dos testes somente o compressor e o tubo capilar foram modificados no equipamento. Os ensaios foram realizados sob condições controladas de temperatura e umidade relativa ambiente $\left(30^{\circ} \mathrm{C}\right.$ e $75 \%$, respectivamente).

ALSAAD e HAMMAD (1998) examinaram a utilização de gás liquefeito de petróleo (GLP) com 24,4\% de R290, 56,4\% de R600 e 17,2\% de R600a como um refrigerante de drop-in para refrigeradores domésticos com R12. Os autores reportaram uma temperatura 
do evaporador de $-15^{\circ} \mathrm{C}$ e coeficiente de performance de 3,4 para uma temperatura de condensação de $27^{\circ} \mathrm{C}$ quando GLP é utilizado.

HAMMAD e ALSAAD (1999) investigaram os parâmetros de performance de refrigerador doméstico sem modificações quando $100 \%$ de R290; 75\% de R290, 19,1\% de R600, 5,9\% de R600a; 50\% de R290, 38,3\% de R600, 11,7\% de R600a; ou 25\% de R290, 57,5\% de R600 e 17,5\% de R600a são usados como possíveis alternativas de substituição ao R12. Os autores mostraram que a mistura de hidrocarbonetos com 50\% de R290, 38,3\% de R600 e 11,7\% de R600a é o melhor refrigerante alternativo.

TASHTOUSH et al., (2002) apresentaram um estudo experimental sobre a performance de refrigeradores domésticos por compressão de vapor com novas misturas de hidrocarbonetos/hidrofluorcarbonos como refrigerantes para substituição do CFC12. Os resultados revelaram que a mistura de R600, R290 e HFC134a apresenta excelente performance.

LEE e SU (2002) conduziram um estudo experimental no uso de isobutano em um refrigerador doméstico. Os resultados mostraram que o coeficiente de performance foi comparável com o obtido pelo CFC12 e HCFC22 quando usados como refrigerantes.

AKASH e SAID (2003) reportaram que o GLP apresenta melhor performance que o R12. Os resultados mostraram que uma carga de GLP de 80 gramas apresentou os melhores resultados quando usado no mesmo refrigerador.

SEKHAR et al., (2004) investigaram a mistura refrigerante de HFC134a/hidrocarbonetos em dois sistemas de baixa temperatura (refrigerador doméstico e freezer) e em dois sistemas de média temperatura (vending machine e bottle cooler). Os autores concluíram que a mistura que contém $9 \%$ de misturas de hidrocarbonetos (em massa) possui a melhor performance, resultando em $10-30 \%$ e $5-15 \%$ de redução no consumo de energia no sistemas de média e baixa temperatura, respectivamente.

FATOUH e EL KAFAFY (2006a) avaliaram teoricamente uma mistura de hidrocarbonetos composta de $60 \%$ de R290 e 40\% de R600 como um melhor substituto drop-in para refrigeradores domésticos baseados em HFC-134a sob condições climáticas anormais, subtropicais e tropicais.

\section{MODELAGEM TEÓRICO-COMPUTACIONAL}

Primeiramente foi desenvolvida uma metodologia de análise teórico-computacional do sistema de refrigeração proposto de forma a se obter estimativa do processo de funcionamento do sistema bem como de sua performance. Para tal análise foi utilizado software específico, a saber, REFPROP 6.0 (McLINDEN et al., 1998), de avaliação de propriedades termodinâmicas e termofísicas de fluidos refrigerantes.

Uma análise teórica foi implementada para a utilização do R134a, propano (R290) e as misturas selecionadas de R290/R600a 60\%/40\%, R290/R600a/R134a 40\%/30\%/30\% e R290/R600a 30\%/70\% no ciclo padrão de refrigeração ASHRAE (temperatura de evaporação: $-23,3^{\circ} \mathrm{C}$, temperatura de condensação: $54,4^{\circ} \mathrm{C}$, temperatura de líquido e de sucção: $32,2^{\circ} \mathrm{C}$ ), utilizando as propriedades termodinâmicas do REFPROP 6.0, conforme recomendado por KIM et al. (1998). O coeficiente de performance de sistemas de 
refrigeração comerciais e domésticos é incrementado de 10 a $20 \%$ quando se utilizam misturas de hidrocarbonetos que contenham R600a e R290 (SEKHAR et al., 2004).

Com o objetivo de simular o refrigerador por compressão a vapor, algumas suposições são necessárias. São estas: a) operação em estado estacionário (regime permanente); b) não ocorre perda de pressão nas tubulações, isto é, as alterações nas pressões ocorrem apenas no compressor e no tubo capilar; c) perdas ou ganhos de calor são negligenciadas; e d) o compressor apresenta eficiência volumétrica ideal e eficiência isoentrópica de $75 \%$ (FATOUH e EL KAFAFY, 2006a). A Figura 1 apresenta o modelo de ciclo termodinâmico utilizado na análise teórico-computacional.

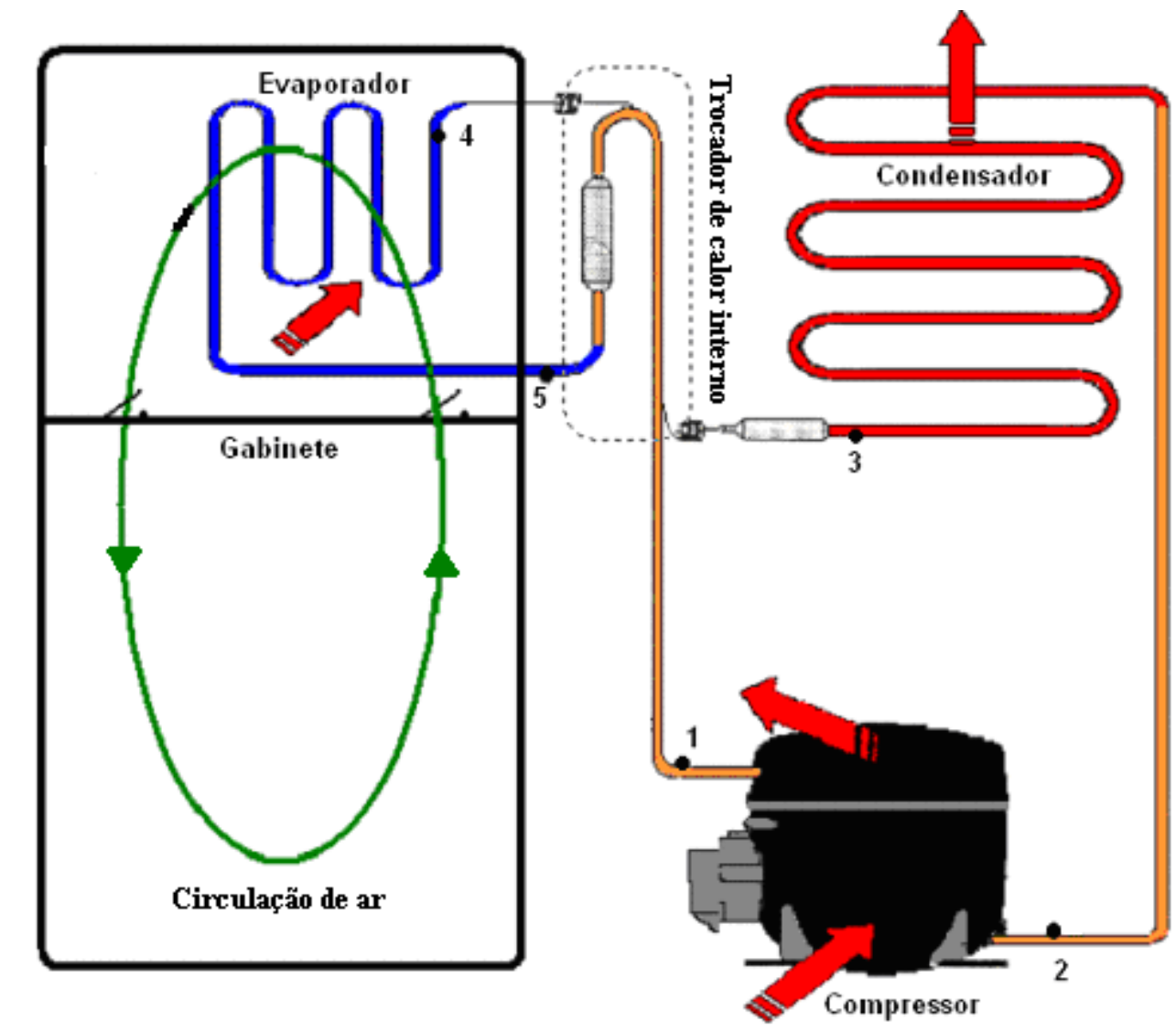

Figura 1 - Ciclo termodinâmico real de um refrigerador doméstico.

(BORGES et al., 2010)

Para a aceitação do drop-in de um fluido de trabalho em um sistema de refrigeração já existente, algumas importantes características de performance devem ser consideradas. São elas: pressões de operação, capacidade de refrigeração volumétrica, coeficiente de performance e temperatura de descarga do compressor (FATOUH e EL KAFAFY, 2006a). O refrigerante deve apresentar um número mínimo de características essenciais favoráveis, dentre as quais as mais significantes são: reduzida densidade na fase líquida, alto calor latente de vaporização, reduzido volume específico na fase vapor e reduzido calor específico na fase líquida. Tais características serão avaliadas e confrontadas entre os fluidos em questão, conforme citado por POGGI et al. (2008).

A capacidade de refrigeração volumétrica $\left(\mathrm{Q}_{\mathrm{vol}}\right)$ é uma medida do tamanho do compressor requerido para condições de operação particulares. Expressa o efeito de refrigeração obtido 
por $1 \mathrm{~m}^{3}$ de refrigerante aspirado pelo compressor (POGGI et al., 2008). Deve-se notar que, à medida que a capacidade volumétrica de refrigeração aumenta, o tamanho do compressor requerido é reduzido. A capacidade de refrigeração volumétrica $\left(\mathrm{Q}_{\mathrm{vol}}\right)$ pode ser estimada como segue:

$$
Q_{v o l}=\frac{\left(h_{5}-h_{4}\right) \cdot \eta_{\text {vol,ideal }}}{v_{1}}
$$

onde $h_{5}$ e $v_{1}$ são, respectivamente, a entalpia específica e o volume específico do refrigerante na condição de vapor saturado à entrada do compressor e $\mathrm{h}_{4}$ é a entalpia específica do refrigerante à entrada do evaporador, de acordo com Fig. 1. A razão de pressão (RP) é definida como a relação entre a pressão de condensação $\left(\mathrm{P}_{\text {cond }}\right)$ e a pressão de evaporação $\left(\mathrm{P}_{\text {evap}}\right)$, isto é,

$$
R P=\frac{P_{\text {cond }}}{P_{\text {evap }}}
$$

As pressões de condensação e evaporação são determinadas de acordo com as temperaturas de condensação e evaporação, respectivamente.

O coeficiente de performance (COP) relaciona a capacidade de refrigeração com a potência requerida e indica o consumo de potência global para uma carga desejada. Alto COP significa baixo consumo de energia para absorção da mesma capacidade de refrigeração do espaço a ser refrigerado. Pode ser expresso como

$$
C O P=\frac{Q_{\text {evap }}}{P_{\text {comp }}}
$$

onde $\mathrm{Q}_{\text {evap }}$ é a capacidade de refrigeração e $\mathrm{P}_{\text {comp }}$ é potência requerida para acionar o compressor. $\mathrm{O}$ balanço de energia no evaporador resulta em:

$$
Q_{\text {evap }}=m_{r} \cdot\left(h_{5}-h_{4}\right)
$$

A potência requerida para acionar o compressor pode ser descrita como:

$$
P_{\text {comp }}=m_{r} \cdot\left(h_{2}-h_{1}\right)
$$

A entalpia específica real do vapor refrigerante superaquecido à saída do compressor $\left(\mathrm{h}_{2}\right)$ pode ser calculada como segue:

$$
h_{2}=h_{1}+\frac{\left(h_{2, i s}-h_{1}\right)}{\eta_{i s, c o m p}}
$$

onde $\eta_{\text {is,comp }}$ é a eficiência isoentrópica do compressor e $h_{2, i s}$ é a entalpia do vapor refrigerante superaquecido à saída do compressor para um processo de compressão isoentrópico. 
A vazão mássica de refrigerante $\left(\mathrm{m}_{\mathrm{r}}\right)$ pode ser estimada utilizando a equação seguinte (TASHTOUSH et al., 2002):

$$
m_{r}=\frac{Q_{\text {evap }}}{q_{\text {evap }}}
$$

Onde $\mathrm{Q}_{\text {evap }}$ é a capacidade de refrigeração em $\mathrm{kW}$ e $\mathrm{q}_{\mathrm{evap}} \mathrm{o}$ efeito de refrigeração específico em kJ/kg. Os dados de vazão mássica na simulação computacional são fornecidos em kg/h.

A temperatura de descarga do compressor $\left(\mathrm{T}_{\mathrm{des}}=\mathrm{T}_{2}\right)$ é um importante parâmetro, devido ao seu efeito sobre os componentes do compressor e a estabilidade dos lubrificantes. Esta temperatura foi determinada utilizando ambos os dados de pressão de condensação e entalpia específica real à saída do compressor, determinada pela Eq.06.

$\mathrm{Na}$ análise do ciclo, a mesma capacidade de refrigeração foi aplicada para todos os refrigerantes considerados. A capacidade de refrigeração de $143 \mathrm{~W}$ foi obtida através da conversão da capacidade de congelamento de um refrigerador doméstico de 210 L, fornecida pelo fabricante, que é de $3,5 \mathrm{~kg} / 24 \mathrm{~h}$.

\section{RESULTADOS E DISCUSSÕES}

Aplicando as diversas equações relativas ao circuito de refrigeração representado na Fig.1 sob as condições previamente definidas de operação (ciclo ASHRAE) e com o uso do software REFPROP 6.0 (McLINDEN et al., 1998) obtiveram-se os dados operacionais referentes aos refrigerantes selecionados. A Tabela 1 apresenta os resultados das simulações. 
SILVA et al (2010)

Tabela 1: Características dos hidrocarbonetos no ciclo de refrigeração padrão

ASHRAE.

\begin{tabular}{|c|c|c|c|c|c|}
\hline Rarâmetros & R134a & $\mathbf{R} 290$ & $\begin{array}{c}\text { R290/R600a } \\
(60 / 40)\end{array}$ & $\begin{array}{c}\text { R290/R600a } \\
(30 / 70)\end{array}$ & $\begin{array}{c}\mathrm{R} 290 / \mathrm{R} 600 \mathrm{a} / \mathrm{R} 134 \mathrm{a} \\
(\mathbf{4 0 / 3 0 / 3 0 )}\end{array}$ \\
\hline $\begin{array}{c}\text { Pressão de } \\
\text { condensação @ } \\
54,4^{\circ} \mathrm{C}(\mathrm{kPa})\end{array}$ & 1470,0 & 1883,0 & 1370,5 & 1050,6 & 1604,0 \\
\hline $\begin{array}{c}\text { Pressão de } \\
\text { evaporação @ } \\
-23,3^{\circ} \mathrm{C} \\
(\mathrm{kPa})\end{array}$ & 114,8 & 216,6 & 140,6 & 99,85 & 174,7 \\
\hline $\begin{array}{c}\text { Diferença de pressão } \\
(\mathrm{kPa})\end{array}$ & 1355,2 & 1666,4 & 1229,9 & 950,75 & 1429,3 \\
\hline Razão de pressões & 12,8 & 8,7 & 9,74 & 10,52 & 9,18 \\
\hline $\begin{array}{c}\text { Efeito de } \\
\text { refrigeração }(\mathrm{kJ} / \mathrm{kg})\end{array}$ & 185,58 & 354,47 & 347,85 & 342,11 & 279,7 \\
\hline $\begin{array}{c}\text { Capacidade de } \\
\text { refrigeração } \\
\text { volumétrica }\left(\mathrm{kJ} / \mathrm{m}^{3}\right)\end{array}$ & 743,719 & 1171,9 & 819,718 & 619,5 & 980,391 \\
\hline $\mathrm{COP}$ & 2,049 & 2,041 & 2,093 & 2,160 & 2,085 \\
\hline $\begin{array}{l}\text { Capacidade de } \\
\text { refrigeração }(\mathrm{W})\end{array}$ & 143 & 143 & 143 & 143 & 143 \\
\hline $\begin{array}{c}\text { Vazão mássica } \\
(\mathrm{kg} / \mathrm{h})\end{array}$ & 2,773 & 1,452 & 1,479 & 1,504 & 1,840 \\
\hline $\begin{array}{l}\text { Temperatura de } \\
\text { descarga }\left({ }^{\circ} \mathrm{C}\right)\end{array}$ & 139,5 & 134,5 & 128,8 & 123,6 & 126,0 \\
\hline $\begin{array}{c}\text { Temperatura de } \\
\text { entrada no tubo } \\
\text { capilar }\left({ }^{\circ} \mathrm{C}\right)\end{array}$ & 32,2 & 32,2 & 32,2 & 32,2 & 32,2 \\
\hline $\begin{array}{c}\text { Volume específico } \\
\text { na sucção } \\
\left(\mathrm{m}^{3} / \mathrm{kg}\right)\end{array}$ & 0,2121 & 0,2571 & 0,3607 & 0,4694 & 0,2425 \\
\hline $\begin{array}{c}\text { Calor específico na } \\
\text { fase líquida } \\
(\mathrm{kJ} / \mathrm{kg} . \mathrm{K})\end{array}$ & 1,44733 & 2,8002 & 2,66510 & 2,57641 & 2,28916 \\
\hline $\begin{array}{c}\text { Densidade na fase } \\
\text { líquida }\left(\mathrm{kg} / \mathrm{m}^{3}\right)\end{array}$ & 1183,27 & 483,44 & 505,718 & 523,598 & 588,688 \\
\hline
\end{tabular}

Analisando os resultados apresentados na Tabela 1 observa-se que, quanto maior a fração em massa de propano (R290), maior será a pressão de condensação e menor a temperatura de evaporação, o que pode acarretar em grande formação de gelo no evaporador. A mistura de R290/R600a (60/40) aponta para a melhor solução neste quesito.

A mistura de R290/R600a (60/40) apresenta as menores densidades de líquido juntamente com o R290 puro, proporcionando assim reduzidas perdas por atrito no sistema. Esta mistura também apresenta as menores densidades na fase vapor e líquida, sendo esperada uma redução no trabalho total de compressão. A redução nos valores de densidades é mais 
importante que um aumento no calor latente de vaporização do fluido (POGGI et al., 2008).

A diminuição da proporção de R134a nas misturas acarreta uma redução da potência de compressão necessária e da transferência de calor no condensador. A mistura de hidrocarbonetos com o R134a proporciona um aumento na lubricidade e miscibilidade do óleo lubrificante com o R134a (TASHTOUSH et al., 2002). As densidades de vapor nas condições de sucção das misturas e do R290 puro se apresentam menores que as do R134a, correspondendo a uma menor carga de massa necessária destes fluidos no sistema em relação ao R134a.

Pode-se notar ainda que o calor latente de vaporização (efeito de refrigeração) da mistura R290/R600a é cerca de duas vezes maior que do R134a. No entanto, devido ao reduzido volume específico do R134a na sucção, as capacidades de refrigeração volumétrica dos dois fluidos são próximas. De modo a aceitar um fluido de trabalho como substituto para um sistema de refrigeração doméstico, uma similar capacidade de refrigeração volumétrica e performance comparada com o refrigerante existente são requeridas (FATOUH e KAFAFY, 2006a).

\section{CONCLUSÕES}

Os esforços de pesquisa e desenvolvimento na área de Refrigeração e Ar-Condicionado aplicados ao uso de fluidos refrigerantes oriundos de fontes naturais não está associada somente à necessidade de preservação do meio ambiente em si, mas também apresenta grande importância devido a necessidade latente do aumento da eficiência energética dos equipamentos.

Hidrocarbonetos, como o gás liquefeito de petróleo (GLP), são ambientalmente corretos e encontram-se disponíveis na natureza, neste sentido, a utilização destas substâncias como fluidos refrigerantes em refrigeradores domésticos é muito atrativa.

De acordo com a análise termodinâmica desenvolvida para os fluidos hidrocarbonetos pode-se observar que estes reduzem os níveis de pressão desenvolvidas no condensador e evaporador.

A utilização do R290 e misturas envolvendo hidrocarbonetos proporciona uma triplicação do calor latente de vaporização em relação ao R134a. Tal fator acarreta uma redução de cerca de $50 \%$ da necessidade de carga em massa de refrigerante no sistema de refrigeração para uma mesma capacidade do equipamento.

Os hidrocarbonetos apresentam menores temperaturas de descarga no compressor, proporcionando maior vida útil a estes componentes. O coeficiente de performance do sistema com hidrocarbonetos e misturas apresentou crescimento de cerca de 5\% em relação ao R134a. Consequentemente, menores trabalhos de compressão são requeridos para os hidrocarbonetos em relação ao R134a devido a suas propriedades termofísicas relativas à densidade na fase líquida e vapor.

De forma a consolidar os hidrocarbonetos e suas misturas como substitutos aos fluidos sintéticos, como o HFC134a, a indústria de refrigeração deve focar seus esforços no desenvolvimento de compressores adequados à capacidade de refrigeração volumétrica dos 
fluidos oriundos de fontes naturais, além do desenvolvimento de novas metodologias de projeto de trocadores de calor, já que as misturas se comportam de forma diferente dos fluidos puros no que diz respeito à mudança de fase.

Como continuidade do projeto de pesquisa serão desenvolvidas análises experimentais destas misturas selecionadas de modo a se obter um indicativo de performance em relação ao HFC-134a, tendo como objeto de comparação os resultados da análise teóricocomputacional.

\section{AGRADECIMENTOS}

Agradecemos à Pró-Reitoria de Pesquisa e Inovação do IFRN pela concessão de bolsas de iniciação científica, pesquisador e verba para apoio ao desenvolvimento de projetos através do Edital No 23/2010.

\section{REFERENCIAS}

1. AKASH, B.A., SAID, S.A., Assessment of LPG as a possible alternative to R-12 in domestic refrigerators. Energy Conversion and Management 44 (2003) 381-388.

2. BORGES, B., MELO, C., GONÇALVES, J., HERMES, C., Simulação Transiente de Refrigeradores Domésticos: Uma abordagem quase-estática semi-empírica. Anais do VI Congresso Nacional de Engenharia Mecânica. Campina Grande - PB. Associação Brasileira de Engenharia e Ciências Mecânicas. 2010.

3. FATOUH, M., EL KAFAFY, M., Assessment of propane/commercial butane mixtures as possible alternatives to R134a in domestic refrigerators. Energy Conversion and Management 47 (2006a) 2644- 2658.

4. FATOUH, M., EL KAFAFY, M., Experimental evaluation of a domestic refrigerator working with LPG. Applied Thermal Engineering 26 (2006b) 1593-1603.

5. HAMMAD, M.A., ALSAAD, M.A., The use of hydrocarbon mixtures as refrigerants in domestic refrigerators. Applied Thermal Engineering 19 (1999) 1181-1189.

6. KIM, M.H., LIM, B.H., CHU, E.S., The performance analysis of a hydrocarbon refrigerant R-600a in a househould refrigerator/freezer. KSME International Journal, Vol.12, No.4, pp.753-760. 1998.

7. LEE, Y.S., SU, C.C., Experimental studies of isobutane (R600a) as the refrigerant in domestic refrigeration system. Applied Thermal Engineering 22 (2002) 507-519.

8. MOHANRAJ, M., JAYARAJ, S., MURALEEDHARAN, C. Improved energy efficiency for HFC134a domestic refrigerator retrofitted with hydrocarbon mixture (HC290/HC600a) as drop-in substitute. Energy for Sustainable Development Volume XI, No.4, December 2007.

9. MACLAINE-CROSS, I.L., Why hydrocarbons save energy. AIRAH Journal 51, pp. 33- 37.

10. MEYERS, S., McMAHON, J.E., McNEIL, M., LIU, X., Impacts of US federal energy efficiency standards for residential appliances. Energy 28 (2003), 755-767. 
11. MELO, C.A., JANNUZZI, G.M., O estoque de refrigeradores no Brasil: diferenças e semelhanças regionais por faixas de renda. Espaço Energia, Número 08, Abril 2008, ISSN: $1807-8575$.

12. McLINDEN, M.O., KLEIN, S.A. LEMMON, E.W. and PESKIN, A.P., REFPROP, Version 6.0, NIST Properties of Refrigerants and Refrigerant Mixtures, NIST Standard Reference Database 23, U.S. Dept. of Commerce, Standard Reference Data Program, Gaithersburg, MD, (1998).

13. POGGI, F., MACCHI-TEJEDA, H., LEDUCQ, D., BONTEMPS, A., Refrigerant charge in refrigeration systems and strategies of charge reduction. International Journal of Refrigeration 31 (2008) 353-370.

14. RICHARDSON, R.N., BUTTERWORTH, J.S., The performance of propane/isobutane mixtures in a vapourcompression refrigeration system. International Journal of Refrigeration 18 (1995), Number 1, 58-62.

15. SEKHAR, S.J., LAL, D.M., HFC134a/HC600a/HC290 mixture a retrofit for CFC12 system. International Journal of Refrigeration 28 (2004), 735-743.

16. TASHTOUSH, B., TAHAT, M., SHUDEIFAT, M.A., Experimental study of new refrigerant mixtures to replace R12 in domestic refrigerators. Applied Thermal Engineering 22 (2002) 495-506.

17. VAN BUSKIRK, R., HAGAN, E.B., AHENKORAH, A.O., McNEIL, M.A., Refrigerator efficiency in Ghana: Tailoring an appliance market transformation program design for Africa. Energy Policy 35 (2007) 2401-2411. 